\title{
BMJ Open Risk of obstructive sleep apnoea in patients with rheumatoid arthritis: a nationwide population-based retrospective cohort study
}

\author{
Te-Chun Shen, ${ }^{1,2,3}$ Liang-Wen Hang, ${ }^{2,4}$ Shinn-Jye Liang, ${ }^{2,4}$ Chien-Chung Huang, ${ }^{1,5}$ \\ Cheng-Li Lin, ${ }^{6}$ Chih-Yen Tu, ${ }^{2}$ Te-Chun Hsia, ${ }^{2}$ Chuen-Ming Shih, ${ }^{2}$ Wu-Huei Hsu, ${ }^{2}$ \\ Fung-Chang Sung $6,7,8$
}

To cite: Shen T-C, Hang L-W, Liang S-J, et al. Risk of obstructive sleep apnoea in patients with rheumatoid arthritis: a nationwide population-based retrospective cohort study. BMJ Open 2016;6:e013151. doi:10.1136/bmjopen-2016013151

- Prepublication history for this paper is available online. To view these files please visit the journal online (http://dx.doi.org/10.1136/ bmjopen-2016-013151)

L-WH and F-CS contributed equally.

Received 23 June 2016 Revised 27 October 2016 Accepted 28 October 2016

CrossMark

For numbered affiliations see end of article.

Correspondence to Professor Fung-Chang Sung; fcsung.tw@gmail.com

\section{ABSTRACT}

Objective: Sleep disorders are prevalent medical disorders in patients with rheumatoid arthritis (RA). However, whether patients with RA are at an increased risk of developing obstructive sleep apnoea (OSA) is unclear.

Design: Using population-based retrospective cohort study to examine the risk of OSA in patients with RA. Setting: We used claims data of the National Health Insurance Research Database (NHIRD) of Taiwan.

Participants: We identified a RA cohort with 33418 patients newly diagnosed in 2000-2010 and a randomly selected non-RA comparison cohort with 33418 individuals frequency matched by sex, age and diagnosis year.

Primary and secondary outcome measures: Incident OSA was estimated by the end of 2011. The HRs of OSA were calculated using the Cox proportional hazards regression analysis.

Results: The overall incidence rate of OSA was 75\% greater in the RA cohort than in the non-RA cohort ( 3.04 vs $1.73 / 10000$ person-years, $p<0.001$ ), with an adjusted HR (aHR) of $1.75(95 \% \mathrm{Cl} 1.18$ to 2.60$)$. Stratified analyses by sex, age group and comorbidity revealed that the incidence rates of OSA associated with RA were higher in all subgroups.

Conclusions: This population-based retrospective cohort study suggested that patients with RA should be monitored for the risk of developing OSA.

\section{INTRODUCTION}

Rheumatoid arthritis (RA) is the most prevalent autoimmune inflammatory arthritis found in adults, with the worldwide prevalence ranging from $0.4 \%$ to $1.3 \% .{ }^{12}$ Patients with the disease, living with decreased quality of life, are at an increased risk of disability and premature death. ${ }^{2}{ }^{3}$ The aetiology of RA is largely unknown and it may result from an interaction between genetic factors and environmental exposures. ${ }^{4}$ The medical diagnosis

\section{Strengths and limitations of this study}

- This is the first population-based retrospective cohort study to examine the risk of obstructive sleep apnoea (OSA) in patients with rheumatoid arthritis (RA).

- The study cohorts were large enough to observe the risk variations among subgroups.

- Information on potential confounding factors, such as body mass index, family history and drink and smoking habits was unavailable.

- Information on the severity of RA and OSA was unavailable to demonstrate dose-response relationship between RA and OSA.

- Relevant clinical variables such as image reports, serum laboratory data and polysomnography results were unavailable to differentiate whether clinical characteristics are associated with the risk of developing OSA.

and therapy for RA usually follows wellestablished clinical practice guidelines. ${ }^{5} 6$

Obstructive sleep apnoea (OSA) is a common but underestimated disorder, which may affect $2-4 \%$ of the adult population. Individuals with OSA are characterised with apnoeas, hypopnoeas or respiratory effort related arousals, occurring for at least five times/hour during sleep (apnoea-hypopnoea index, AHI $\geq 5$ ). ${ }^{7}$ Patients may suffer from loud snoring and breathing interruptions leading to daytime sleepiness. Men, old age, obesity and sometimes, orthopaedic pathology are risk factors associated with OSA. ${ }^{8-10}$ This disorder has been, in particular, associated with the development of cardiovascular comorbidities and even with sudden death. ${ }^{11}$

Evidence has shown the association between RA and subsequent OSA. An earlier case report in 1983 showed a patient with RA 
related adult-acquired micrognathia could develop severe OSA. ${ }^{12}$ Subsequently, several case studies also reported an increased risk of OSA in patients with RA with cervical or temporomandibular lesions. ${ }^{13-20}$ It is interesting to note that Gjevre $e t a l^{21}$ have even found a very high prevalence of OSA in patients with RA: 8 OSA cases in 10 patients with RA $(80 \%)$ with abnormal Epworth sleepiness scale (ESS) scores $(\geq 10)$, and 9 OSA cases in 15 patients with RA $(60 \%)$ with normal ESS scores $(<10)$. Furthermore, Mutoh et $a t^{22}$ recently reported that among 62 hospitalised patients with RA, 23 cases $(37 \%)$ had been confirmed to have OSA,and 13 patients $(20.9 \%)$ were moderate-to-severe cases. In addition, Reading et $a l^{23}$ conducted a sex and agematched comparative study and found a higher OSA prevalence in patients with RA $(23 / 164,14 \%)$ than in non-RA controls $(30 / 328,9.1 \%)(\mathrm{p}=0.13)$.

Previous studies on the relationship between RA and OSA risk were limited to case reports and case-control studies with small sample sizes. No large scale population-based follow-up study has been performed. This study used the National Health Insurance Research Database (NHIRD) of Taiwan to conduct a retrospective cohort analysis to compare the OSA risk between patients with RA and without RA. NHIRD is a reliable data source and has been used in various studies, including studies on issues of RA and OSA. ${ }^{24-27}$

\section{MATERIALS AND METHODS}

\section{Data source}

This cohort study used reimbursement claims data from the Longitudinal Health Insurance Database for Catastrophic Illness Patients (LHID-CIP), which is part of the NHIRD used for research purposes. The National Health Insurance (NHI) programme is a mandatory universal health insurance system covering more than $99 \%$ of Taiwan's 23.74 million population (http://nhird.nhri. org.tw/en/index.html). The NHIRD database contains information on the demographic status of insured population, diagnostic codes and care and medications for inpatients and outpatients. Advantages of using NHIRD in research have been previously described. ${ }^{28}$ The National Health Research Institute (NHRI) manages the NHIRD, and provides scrambled random identification numbers to ensure patient privacy.

\section{Study cohorts}

We used LHID-CIP file and identified 33418 patients who were newly diagnosed with RA (International Classification of Diseases Ninth Edition Clinical Modification (ICD-9-CM) code 714) between 2000 and 2010 as the RA cohort. Individuals with a history of OSA or those $<20$ years were excluded. Thus, we established the non-RA cohort with those free of RA and OSA, frequency matched by sex, age and diagnosis year of patients with RA. Both cohorts were followed up until OSA was diagnosed, withdrawal from the insurance programme, death or until the end of 2011. The diagnostic criteria of OSA was that people with a diagnosis of OSA (ICD-9-CM codes 327.23, 780.51, 780.53 and 780.57) following a diagnostic examination of polysomnography (PSG, examination codes: 17008A and 17008B in NHIRD). Selected comorbidities were identified at the baseline, including hypertension (ICD-9-CM codes 401-405), diabetes (ICD-9-CM code 250), hyperlipidaemia (ICD-9-CM code 272), ischaemic heart disease (IHD) (ICD-9-CM codes 410-414), dysrhythmia (ICD-9-CM code 427), congestive heart failure (CHF) (ICD-9-CM code 428), stroke (ICD-9-CM codes 430 -438), chronic kidney disease (CKD) (ICD-9-CM code 585), asthma/chronic obstructive pulmonary disease (COPD) (ICD-9-CM codes 493, 496) and obesity (ICD-9-CM code 278.0).

\section{Statistical analysis}

The $\chi^{2}$ test was used to compare the categorical distributions of sex, age and baseline comorbidities between the RA and the non-RA cohorts. Differences in the continuous variables between the two cohorts were examined using the Student's t-test. Cumulative incidence curves of OSA for both cohorts were plotted using the Kaplan-Meier method, and the differences in curves between the two cohorts were examined using the log-rank test. The OSA incidence densities were estimated by dividing the number of OSA cases by the follow-up person-years for both cohorts and by covariates. Univariable and multivariable Cox proportion hazards regression models were employed to examine the RA cohort to non-RA cohort crude HRs (cHRs) and adjusted HRs (aHRs) of OSA, respectively, and related 95\% CIs. Covariates used in the multivariable model included sex, age and comorbidities of hypertension, diabetes, hyperlipidaemia, IHD, dysrhythmia, CHF, stroke, CKD, asthma/COPD and obesity. All analyses were conducted using SAS V.9.4 (SAS Institute, Cary, North Carolina, USA), with the significance level set as 0.05 in the two-tailed tests.

\section{RESULTS}

Both cohorts had similar distributions for sex and age, and were found to be predominant in women $(77.6 \%)$, and in the age group of 20-49 years (39.0\%; table 1 ). The mean ages of the RA and the non-RA cohorts were $54.0(\mathrm{SD}=13.9)$, and $53.5(\mathrm{SD}=14.3)$ years, respectively. As compared with the non-RA cohort, the RA cohort exhibited a higher prevalence in the comorbidities of hypertension, IHD, dysrhythmia, CHF, stroke, CKD, and asthma/COPD at the baseline $(p<0.05)$. During the mean follow-up period of $6.62 \pm 3.62$ years for the RA cohort and $6.75 \pm 3.63$ years for the non-RA cohort, the results of the Kaplan-Meier analysis revealed that the RA cohort exhibited a higher cumulative incidence of OSA than the non-RA cohort (log-rank test, $\mathrm{p}=0.005$; figure 1). 
Table 1 Baseline characteristics of individuals compared between RA and non-RA cohorts

\begin{tabular}{|c|c|c|c|c|c|}
\hline & \multicolumn{4}{|l|}{ RA } & \multirow[b]{3}{*}{ p Value } \\
\hline & \multicolumn{2}{|c|}{ Yes $(\mathrm{N}=33$ 418) } & \multicolumn{2}{|c|}{ No $(\mathrm{N}=33$ 418) } & \\
\hline & $\bar{n}$ & Per cent & $\bar{n}$ & Per cent & \\
\hline Age, year & & & & & 0.99 \\
\hline $20-49$ & 13034 & 39.0 & 13034 & 39.0 & \\
\hline $50-64$ & 12604 & 37.7 & 12604 & 37.7 & \\
\hline$\geq 65$ & 7780 & 23.3 & 7780 & 23.3 & \\
\hline Mean $\pm S D^{*}$ & 54.0 & \pm 13.9 & 53.5 & \pm 14.3 & $<0.001$ \\
\hline Sex & & & & & 0.99 \\
\hline Female & 25943 & 77.6 & 25943 & 77.6 & \\
\hline Male & 7475 & 22.4 & 7475 & 22.4 & \\
\hline \multicolumn{6}{|l|}{ Comorbidity } \\
\hline Hypertension & 10957 & 32.8 & 10496 & 31.4 & 0.001 \\
\hline Diabetes & 2900 & 8.68 & 2852 & 8.53 & 0.51 \\
\hline Hyperlipidaemia & 6724 & 20.1 & 6724 & 20.1 & 0.99 \\
\hline IHD & 5471 & 16.4 & 4966 & 14.9 & $<0.001$ \\
\hline Dysrhythmia & 2638 & 7.89 & 2080 & 6.22 & $<0.001$ \\
\hline $\mathrm{CHF}$ & 1176 & 3.52 & 815 & 2.44 & $<0.001$ \\
\hline Stroke & 938 & 2.81 & 1066 & 3.19 & 0.004 \\
\hline CKD & 428 & 1.28 & 677 & 2.03 & $<0.001$ \\
\hline Asthma/COPD & 4154 & 12.4 & 2738 & 8.19 & $<0.001$ \\
\hline Obesity & 455 & 1.36 & 447 & 1.34 & 0.79 \\
\hline
\end{tabular}

The overall incidences of OSA, in the RA and the non-RA cohorts, were 3.04 and 1.73/10 000 person-years, respectively. After adjustment for sex, age and comorbidities, patients with RA exhibited an increased risk of OSA as compared with those without RA (adjusted HR (aHR) $=1.75,95 \%$ CI 1.18 to 2.60 ) (table 2). The incidence rates of OSA were higher in men than in women (5.75 and $1.50 / 10000$ person-years) and in patients with any selected comorbidity than in those without the comorbidity. In the multivariable model analyses, the risk of OSA was 4.19-fold higher in men than in women (95\% CI 2.85 to 6.15) and was higher in patients with hyperlipidaemia, IHD and obesity $(p<0.05)$.

Table 3 shows the OSA incidence rates for both RA and non-RA cohorts and the RA cohort to non-RA cohort HRs by sex, age and comorbidity. Men had higher incidence of OSA than women had in both cohorts, and sex-specific RA cohort to non-RA cohort aHRs of OSA were 2.00 for men (95\% CI 1.13 to 3.54) and 1.51 for women (95\% CI 0.87 to 2.62). The incidence of OSA was higher in patients with any comorbidity than in those without any comorbidity in both cohorts. In the RA cohort, the incident OSA was 2.52-fold higher in patients with comorbidity than those without the comorbidity.

\section{DISCUSSION}

To the best of our knowledge, this is the first retrospective cohort study using nationwide population-based data

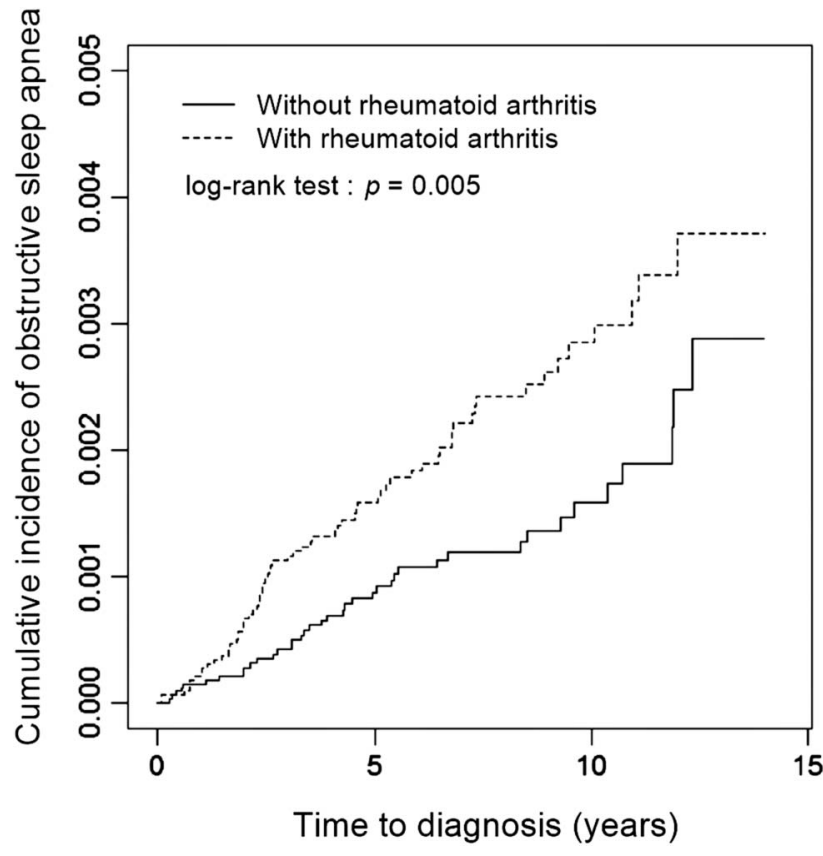

Figure 1 Cumulative incidence of obstructive sleep apnoea in cohorts with and without rheumatoid arthritis.

to evaluate the OSA risk associating with RA. Results show a $75 \%$ greater hazard of OSA for patients with RA compared with those without RA. The OSA risk was greater in men than in women, in old people than in young people and in individuals with comorbidity than 
Table 2 Incidence rates and HRs of obstructive sleep apnoea in study population by study cohort and covariates

\begin{tabular}{|c|c|c|c|c|c|}
\hline Variable & Event & PY & Rate† & Relative HR $(95 \% \mathrm{Cl})$ & Adjusted HR $(95 \% \mathrm{Cl})$ \\
\hline \multicolumn{6}{|l|}{$\mathrm{RA}$} \\
\hline No & 39 & 225165 & 1.73 & 1.00 & 1.00 \\
\hline Yes & 67 & 220536 & 3.04 & $1.75(1.18 \text { to } 2.60)^{\star * \star}$ & $1.75(1.18 \text { to } 2.60)^{\star \star \star}$ \\
\hline \multicolumn{6}{|l|}{ Age, year } \\
\hline $20-49$ & 42 & 186857 & 2.25 & 1.00 & $1.99(1.09 \text { to } 3.65)^{*}$ \\
\hline $50-64$ & 44 & 170349 & 2.58 & $1.15(0.75$ to 1.75$)$ & 1.64 (0.95 to 2.83$)$ \\
\hline$\geq 65$ & 20 & 88493 & 2.26 & $1.00(0.59$ to 1.71$)$ & 1.00 \\
\hline \multicolumn{6}{|l|}{ Sex } \\
\hline Female & 53 & 353496 & 1.50 & 1.00 & 1.00 \\
\hline Male & 53 & 92205 & 5.75 & $3.84(2.62 \text { to } 5.62)^{\star \star \star}$ & $4.19(2.85 \text { to } 6.15)^{\star \star \star}$ \\
\hline \multicolumn{6}{|c|}{ Comorbidity } \\
\hline \multicolumn{6}{|c|}{ Hypertension } \\
\hline No & 63 & 314159 & 2.01 & 1.00 & 1.00 \\
\hline Yes & 43 & 131542 & 3.27 & $1.63(1.11 \text { to } 2.41)^{*}$ & $1.12(0.69$ to 1.80$)$ \\
\hline \multicolumn{6}{|l|}{ Diabetes } \\
\hline No & 95 & 414708 & 2.29 & 1.00 & 1.00 \\
\hline Yes & 11 & 30993 & 3.55 & 1.55 (0.83 to 2.90$)$ & - \\
\hline \multicolumn{6}{|c|}{ Hyperlipidaemia } \\
\hline No & 70 & 364848 & 1.92 & 1.00 & 1.00 \\
\hline Yes & 36 & 80853 & 4.45 & $2.33(1.56 \text { to } 3.48)^{\star \star \star}$ & $1.88(1.19 \text { to } 2.96)^{\star \star}$ \\
\hline \multicolumn{6}{|l|}{ IHD } \\
\hline No & 74 & 383569 & 1.93 & 1.00 & 1.00 \\
\hline Yes & 32 & 62131 & 5.15 & $2.68(1.77 \text { to } 4.07)^{\star \star \star}$ & $2.41(1.47 \text { to } 3.95)^{\star \star \star}$ \\
\hline \multicolumn{6}{|c|}{ Dysrhythmia } \\
\hline No & 96 & 420524 & 2.28 & 1.00 & 1.00 \\
\hline Yes & 10 & 25176 & 3.97 & $1.74(0.91$ to 3.35$)$ & - \\
\hline \multicolumn{6}{|l|}{$\mathrm{CHF}$} \\
\hline No & 102 & 436263 & 2.34 & 1.00 & 1.00 \\
\hline Yes & 4 & 9438 & 4.24 & 1.81 (0.67 to 4.93$)$ & - \\
\hline \multicolumn{6}{|l|}{ Stroke } \\
\hline No & 103 & 435955 & 2.36 & 1.00 & 1.00 \\
\hline Yes & 3 & 9746 & 3.08 & $1.30(0.41$ to 4.11$)$ & - \\
\hline \multicolumn{6}{|l|}{ CKD } \\
\hline No & 103 & 440392 & 2.34 & 1.00 & 1.00 \\
\hline Yes & 3 & 5309 & 5.65 & 2.41 (0.76 to 7.59$)$ & - \\
\hline \multicolumn{6}{|c|}{ Asthma/COPD } \\
\hline No & 92 & 408410 & 2.25 & 1.00 & 1.00 \\
\hline Yes & 14 & 37291 & 3.75 & $1.67(0.95$ to 2.94$)$ & - \\
\hline \multicolumn{6}{|l|}{ Obesity } \\
\hline No & 100 & 441155 & 2.27 & 1.00 & 1.00 \\
\hline Yes & 6 & 4546 & 13.2 & $5.86(2.57 \text { to } 13.4)^{\star \star \star}$ & $5.22(2.24 \text { to } 12.2)^{\star * *}$ \\
\hline
\end{tabular}

${ }^{*} \mathrm{p}<0.05,{ }^{* *} \mathrm{p}<0.01,{ }^{* * *} \mathrm{p}<0.001$.

†Incidence rate per 10000 person-years.

¥Multivariable analysis including sex, age and comorbidities of hypertension, diabetes, hyperlipidemia, IHD, dysrhythmia, CHF, stroke, CKD, asthma/COPD and obesity.

$\mathrm{CHF}$, congestive heart failure; CKD, chronic kidney disease; COPD, chronic obstructive pulmonary disease; IHD, ischemic heart disease; PY, person-years; RA, rheumatoid arthritis.

in those without comorbidity. These findings are in accordance with the generally acknowledged concepts. Furthermore, we provided detailed epidemiological characteristics and important comorbid conditions for RA by using the large database available.

In the present study, the risk of OSA has been measured in association with several well-known contributing factors. It is of interest to note that cHRs of OSA are significantly associated with hypertension, hyperlipidaemia, IHD, and obesity in the univariate analysis and the aHRs of OSA remained significant for hyperlipidaemia, IHD and obesity in the multivariate analysis. In addition, most studies to evaluate the risk of OSA were men or equal predominant. This study population, however, was predominantly composed of women (77.6\%). Therefore, the data obtained is valuable because the incidence of OSA in a large female population has less been reported.

To date, the mechanisms explaining the relationship between RA and OSA remain largely unclear. Several 
Table 3 Incidences of obstructive sleep apnoea and RA cohort to non-RA cohort HRs of obstructive sleep apnoea

\begin{tabular}{|c|c|c|c|c|c|c|c|c|}
\hline \multirow[b]{2}{*}{ Variable } & \multicolumn{3}{|l|}{$\mathbf{R A}$} & \multicolumn{3}{|c|}{ Non-RA } & \multirow[b]{2}{*}{ Relative HR (95\% Cl) } & \multirow[b]{2}{*}{ Adjusted HR $¥(95 \% \mathrm{Cl})$} \\
\hline & Event & PY & Rate† & Event & PY & Rate† & & \\
\hline \multicolumn{9}{|l|}{ Sex } \\
\hline Female & 32 & 175312 & 1.83 & 21 & 178183 & 1.18 & 1.55 (0.89 to 2.69$)$ & $1.51(0.87$ to 2.62$)$ \\
\hline Male & 35 & 45223 & 7.74 & 18 & 46982 & 3.83 & $2.02(1.14 \text { to } 3.56)^{*}$ & $2.00(1.13 \text { to } 3.54)^{\star}$ \\
\hline \multicolumn{9}{|l|}{ Age, year } \\
\hline $20-49$ & 25 & 93965 & 2.66 & 17 & 92892 & 1.83 & 1.45 (0.78 to 2.68$)$ & $1.39(0.75$ to 2.58$)$ \\
\hline $50-64$ & 28 & 84234 & 3.32 & 16 & 86115 & 1.86 & 1.79 (0.97 to 3.30$)$ & 1.81 (0.98 to 3.36$)$ \\
\hline$\geq 65$ & 14 & 42336 & 3.31 & 6 & 46157 & 1.30 & $2.44(0.94$ to 6.34$)$ & $2.66(1.01 \text { to } 6.97)^{*}$ \\
\hline \multicolumn{9}{|c|}{ Comorbidity§ } \\
\hline No & 21 & 118101 & 1.78 & 15 & 131128 & 1.14 & $1.55(0.80$ to 3.01$)$ & $1.58(0.81$ to 3.06$)$ \\
\hline Yes & 46 & 102434 & 4.49 & 24 & 94037 & 2.55 & $1.76(1.07 \text { to } 2.88)^{*}$ & $1.66(1.01 \text { to } 2.73)^{\star}$ \\
\hline
\end{tabular}

${ }^{*} \mathrm{p}<0.05$.

†Incidence rate per 10000 person-years.

¥Multivariable analysis including sex, age and comorbidities of hypertension, diabetes, hyperlipidemia, IHD, dysrhythmia, CHF, stroke, CKD, asthma/COPD and obesity.

§Patients with any comorbidity of hypertension, diabetes, hyperlipidaemia, IHD, dysrhythmia, CHF, stroke, CKD, asthma/COPD and obesity were classified into the comorbidity group.

$\mathrm{CHF}$, congestive heart failure; CKD, chronic kidney disease; COPD, chronic obstructive pulmonary disease; IHD, ischemic heart disease; PY, person-years; RA, rheumatoid arthritis.

clinical features of RA have been recognised as contributing factors for OSA, such as micrognathia, cervical spine pathology, temporomandibular joint involvement, cricoarytenoid joint involvement and obesity. ${ }^{21}$ In addition, genetic factors, environmental exposures, shared comorbidities and inadequate lifestyles such as fat and sugar-heavy diet, alcohol consumption, heavy smoking or lack of exercise may contribute to the occurrence of OSA in patients with RA.

It has been acknowledged that patients with RA are at an increased risk of unrecognised cardiovascular diseases and sudden deaths. ${ }^{29}$ In fact, OSA also plays an important role in the increased risk of cardiovascular diseases. OSA can affect autonomic arousal and these autonomic abnormalities may occur in patients with OSA even when they are awake and not hypoxic. In addition, OSA has been linked to inflammation, coagulation and endothelial dysfunction. ${ }^{30}$ Therefore, early diagnosis and adequate treatment for OSA is necessary, which may help eliminate the cardiovascular comorbidities and mortality in patients with RA. ${ }^{31}$

Evidence has shown that patients with RA with cervical and temporomandibular lesions are associated with a much higher risk of OSA (50-100\%). ${ }^{32-34}$ Recently, Mutoh et al confirmed a close association between temporomandibular joint abnormality (TMJA) and OSA; patients with moderate-to-severe OSA had a higher level of TMJA, and the converse is also true. Patients with RA at a higher TMJA stage tend to have more severe OSA. ${ }^{22}$ Therefore, in addition to common strategies to treat OSA there is a need of specific care for patients with RA with orthopaedic pathology. Few studies have reported an improvement of OSA in patients with RA with orthopaedic pathology after receiving surgical treatment. ${ }^{18} 34$

The strength of this study was the use of a nationwide population-based data to evaluate OSA risk in patients with RA. While it is expensive to conduct a population- based prospective cohort study, a retrospective population-based cohort study using insurance data is a suitable and economical alternative. Several limitations should be considered when interpreting our findings. First, we used the ICD-9-CM algorithm to define RA, OSA and comorbidities. However, the diagnosis of RA is reliable because it is classified a catastrophic illness, which requires a carefully peer-reviewed technique to confirm the diagnosis. The diagnosis of OSA was based on both polysomnography and clinical evaluation. The diagnoses of selected comorbidities depended on the competence of clinical physicians. An ad hoc committee, established by the insurance authority, has been actively evaluating the claims data to prevent errors and violation of confidentiality. Moreover, only diseases with at least two diagnoses within 1 year were identified as comorbid disorders, to increase the validity and accuracy of the diagnosis. Second, NHIRD did not provide detailed information on the severity of RA or OSA, and potential confounding factors, such as height, body weight, family history of RA and/or OSA, and lifestyles including drinking and smoking habits. In addition, relevant clinical variables such as image reports, serum laboratory data and PSG result were unavailable in our study. In addition, our estimates of the elevated risk of OSA are likely conservative, due to the requirement for PSG to define OSA. It is likely that many people with OSA do not undergo PSG. Further, because of the higher overall prevalence of OSA in men, it is likely that OSA is disproportionately under-recognised in women.

\section{CONCLUSION}

This population-based cohort study suggests that patients with RA are at an increased risk of developing OSA. Therefore, it is important to evaluate sleep quality and quantity for patients with RA to detect the occurrence of OSA and to reduce further complications. 
Author affiliations

${ }^{1}$ Graduate Institute of Clinical Medicine Science, College of Medicine, China Medical University, Taichung, Taiwan

${ }^{2}$ Department of Internal Medicine, Division of Pulmonary and Critical Care Medicine, China Medical University Hospital, Taichung, Taiwan

${ }^{3}$ Intensive Care Unit, Chu Shang Show Chwan Hospital, Nantau, Taiwan ${ }^{4}$ Sleep Medical Center, China Medical University Hospital, Taichung, Taiwan ${ }^{5}$ Department of Internal Medicine, Division of Immunology and Rheumatology, China Medical University Hospital, Taichung, Taiwan ${ }^{6}$ Management Office for Health Data, China Medical University Hospital, Taichung, Taiwan

${ }^{7}$ Department of Health Services Administration, China Medical University, Taichung, Taiwan

${ }^{8}$ College of Public Health, Kunming Medical University, Kunming, YuanNan, China

Contributors T-CS, L-WH, S-JL and C-CH conceived and designed the study. C-YT, T-CH, C-MS and W-HH provided administrative support. T-CS, L-WH, $\mathrm{C}-\mathrm{LL}$ and F-CS analysed and interpreted the data. T-CS, L-WH, C-LL and F-CS contributed by writing the manuscript. All authors were involved in collection and assembly of data. All authors approved the final version of the manuscript to be published.

Funding This study is supported in part by Taiwan Ministry of Health and Welfare Clinical Trial and Research Center of Excellence (MOHW105-TDU-B-212-133019); Academia Sinica Taiwan Biobank, Stroke Biosignature Project (BM10501010037); NRPB Stroke Clinical Trial Consortium (MOST 105-2325-B-039-003); Tseng-Lien Lin Foundation, Taichung, Taiwan; Taiwan Brain Disease Foundation, Taipei, Taiwan; Katsuzo and Kiyo Aoshima Memorial Funds, Japan; China Medical University Hospital; CMU under the Aim for Top University Plan of the Ministry of Education, Taiwan. The funders had no role in study design, data collection and analysis, decision to publish, or preparation of the manuscript. No additional external funding received for this study.

Competing interests None declared.

Ethics approval This study was approved by the Research Ethics Committee at the China Medical University and Hospital (CMUH-104-REC2-115).

Provenance and peer review Not commissioned; externally peer reviewed.

Data sharing statement No additional data are available.

Open Access This is an Open Access article distributed in accordance with the Creative Commons Attribution Non Commercial (CC BY-NC 4.0) license, which permits others to distribute, remix, adapt, build upon this work noncommercially, and license their derivative works on different terms, provided the original work is properly cited and the use is non-commercial. See: http:// creativecommons.org/licenses/by-nc/4.0/

\section{REFERENCES}

1. Helmick CG, Felson DT, Lawrence RC, et al., National Arthritis Data Workgroup. Estimates of the prevalence of arthritis and other rheumatic conditions in the United States. Part I. Arthritis Rheum 2008;58:15-25.

2. Sacks JJ, Luo YH, Helmick CG. Prevalence of specific types of arthritis and other rheumatic conditions in the ambulatory health care system in the United States, 2001-2005. Arthritis Care Res (Hoboken) 2010;62:460-4.

3. Sihvonen S, Korpela M, Laippala P, et al. Death rates and causes of death in patients with rheumatoid arthritis: a population-based study. Scand J Rheumatol 2004;33:221-7.

4. Symmons DP. Epidemiology of rheumatoid arthritis: determinants of onset, persistence and outcome. Best Pract Res Clin Rheumatol 2002;16:707-22.

5. Aletaha D, Neogi T, Silman AJ, et al. 2010 Rheumatoid arthritis classification criteria: an American College of Rheumatology/ European League Against Rheumatism collaborative initiative. Arthritis Rheum 2010;62:2569-81.

6. Singh JA, Saag KG, Bridges SL Jr, et al. 2015 American College of Rheumatology guideline for the treatment of rheumatoid arthritis. Arthritis Rheumatol 2016;68:1-26.

7. Epstein LJ, Kristo D, Strollo PJ Jr, et al. Clinical guideline for the evaluation, management and long-term care of obstructive sleep apnea in adults. J Clin Sleep Med 2009;5:263-76.
8. Azagra-Calero E, Espinar-Escalona E, Barrera-Mora JM, et al. Obstructive sleep apnea syndrome (OSAS). Review of the literature. Med Oral Patol Oral Cir Bucal 2012;17:e925-9.

9. Young T, Peppard PE, Gottlieb DJ. Epidemiology of obstructive sleep apnea: a population health perspective. Am J Respir Crit Care Med 2002;165:1217-39.

10. Khan A, Than KD, Chen KS, et al. Sleep apnea and cervical spine pathology. Eur Spine J 2014;23:641-7.

11. Weiss JW, Launois SH, Anand A, et al. Cardiovascular morbidity in obstructive sleep apnea. Prog Cardiovasc Dis 1999;41:367-76.

12. Davies SF, Iber C. Obstructive sleep apnea associated with adult-acquired micrognathia from rheumatoid arthritis. Am Rev Respir Dis 1983;127:245-7.

13. Redlund-Johnell I. Upper airway obstruction in patients with rheumatoid arthritis and temporomandibular joint destruction. Scand $J$ Rheumatol 1988;17:273-9.

14. Yoshizawa $\mathrm{H}$, Nagao $\mathrm{M}$, Nakano $\mathrm{M}$, et al. A case of malignant rheumatoid arthritis associated with obstructive sleep apnea due to mandibular lesions. Ryumachi 1991;31:290-7.

15. Sugahara T, Mori $Y$, Kawamoto T, et al. Obstructive sleep apnea associated with temporomandibular joint destruction by rheumatoid arthritis: report of case. J Oral Maxillofac Surg 1994:52:876-80.

16. Izumiyama T, Takahashi M, Sato K, et al. [Relationship between apneic episodes and destruction of temporomandibular joints in patients with rheumatoid arthritis]. Ryumachi 1994;34:761-6.

17. Pépin JL, Della Negra E, Grosclaude S, et al. Sleep apnea syndrome secondary to rheumatoid arthritis. Thorax 1995;50:692-4; discussion 696-7.

18. Bettega G, Pépin JL, Lévy $P$, et al. Surgical treatment of a patient with obstructive sleep apnea syndrome associated with temporomandibular joint destruction by rheumatoid arthritis. Plast Reconstr Surg 1998;101:1045-50.

19. Drossaers-Bakker KW, Hamburger HL, Bongartz EB, et al. Sleep apnea caused by rheumatoid arthritis. Br J Rheumatol 1998;37:889-94.

20. Hamilton J, Dagg K, Sturrock R, et al. Sleep apnea caused by rheumatoid arthritis. Rheumatology (Oxford) 1999;38:679-80.

21. Gjevre JA, Taylor-Gjevre RM, Nair BV, et al. Do sleepy rheumatoid arthritis patients have a sleep disorder? Musculoskeletal Care 2012;10:187-95.

22. Mutoh T, Okuda $Y$, Mokuda $S$, et al. Study on the frequency and risk factors of moderate-to-severe sleep apnea syndrome in rheumatoid arthritis. Mod Rheumatol 2016;26:681-4.

23. Reading SR, Crowson CS, Rodeheffer RJ, et al. Do rheumatoid arthritis patients have a higher risk for sleep apnea? J Rheumatol 2009;36:1869-72.

24. Chung WS, Peng CL, Lin CL, et al. Rheumatoid arthritis increases the risk of deep vein thrombosis and pulmonary thromboembolism: a nationwide cohort study. Ann Rheum Dis 2014;73:1774-80.

25. Lin MC, Guo HR, Lu MC, et al. Increased risk of depression in patients with rheumatoid arthritis: a seven-year population-based cohort study. Clinics (Sao Paulo) 2015;70:91-6.

26. Shen TC, Lin CL, Wei CC, et al. Risk of obstructive sleep apnea in adult patients with asthma: a population-based cohort study in Taiwan. PLOS ONE 2015;10:e0128461.

27. Kao LT, Lee HC, Lin HC, et al. Healthcare service utilization by patients with obstructive sleep apnea: a population-based study. PLOS ONE 2015;10:e0137459.

28. Hsing AW, loannidis JP. Nationwide Population Science: Lessons from the Taiwan National Health Insurance research Database. JAMA Intern Med 2015;175:1527-9.

29. Maradit-Kremers H, Crowson CS, Nicola PJ, et al. Increased unrecognized coronary heart disease and sudden deaths in rheumatoid arthritis: a population-based cohort study. Arthritis Rheum 2005;52:402-11.

30. Holman AJ. Considering cardiovascular mortality in patients with rheumatoid arthritis from a different perspective: a role for autonomic dysregulation and obstructive sleep apnea. $J$ Rheumatol 2007;34:671-3.

31. Hollan I, Dessein PH, Ronda N, et al. Prevention of cardiovascular disease in rheumatoid arthritis. Autoimmun Rev 2015;14:952-69.

32. Oyama T, Okuda $\mathrm{Y}$, Oyama $\mathrm{H}$, et al. [Sleep apnea syndrome in rheumatoid arthritis (RA) patients complicated with cervical and temporomandibular lesions]. Ryumachi 1995;35:3-8.

33. Shoda N, Seichi A, Takeshita K, et al. Sleep apnea in rheumatoid arthritis patients with occipitocervical lesions: the prevalence and associated radiographic features. Eur Spine J 2009;18:905-10.

34. Ataka $\mathrm{H}$, Tanno T, Miyashita $\mathrm{T}$, et al. Occipitocervical fusion has potential to improve sleep apnea in patients with rheumatoid arthritis and upper cervical lesions. Spine 2010;35:E971-5. 